\title{
Toxic Epidermal Necrolysis and Graft-versus-Host Reaction: Revisiting a Puzzling Similarity
}

\author{
G. E. Piérard, ${ }^{1,2}$ T. Hermanns-Lê, ${ }^{3}$ P. Paquet, ${ }^{3}$ A. F. Rousseau, ${ }^{4}$ P. Delvenne, ${ }^{3}$ \\ and C. Piérard-Franchimont ${ }^{1,3}$ \\ ${ }^{1}$ Laboratory of Skin Bioengineering and Imaging (LABIC), Department of Clinical Sciences, University of Liège, \\ 4000 Liège, Belgium \\ ${ }^{2}$ University of Franche-Comté, 25030 Besançon, France \\ ${ }^{3}$ Department of Dermatopathology, Unilab Lg, Liège University Hospital, 4000 Liège, Belgium \\ ${ }^{4}$ Intensive Care Department and Burn Centre, Liège University Hospital, 4000 Liège, Belgium \\ Correspondence should be addressed to G. E. Piérard; gerald.pierard@ulg.ac.be
}

Received 4 April 2013; Accepted 12 May 2013

Academic Editors: E. Alpsoy and B. Amichai

Copyright (C) 2013 G. E. Piérard et al. This is an open access article distributed under the Creative Commons Attribution License, which permits unrestricted use, distribution, and reproduction in any medium, provided the original work is properly cited.

Drug-induced toxic epidermal necrolysis (TEN) and acute cutaneous graft-versus-host reaction (GVHR) under immunopreventive therapy share some histopathological resemblance. So far, there are no serum biomarkers and no immunohistochemical criteria distinguishing with confidence and specificity the skin lesions of TEN and GVHR. Both diseases present as an inflammatory cellpoor necrotic reaction of the epidermis. This report compares three sets of 15 immunostaining patterns found in TEN, GVHR, and partial thickness thermal burns (PTTB), respectively. Three series of 17 skin biopsies were scrutinized. Irrespective of the distinct causal pathobiology of TEN and GVHR, similar secondary effector cells were recruited in lesional skin. Burns were less enriched in cells of the monocyte-macrophage disease. These cells likely exert deleterious effects in TEN and GVHR and cannot be simply regarded as passive bystanders. These life-threatening conditions are probably nursed, at least in part, by macrophages.

\section{Introduction}

Toxic epidermal necrolysis (TEN), formerly called under its eponym Lyell's syndrome, is a severe cutaneous adverse reaction (SCAR) to drugs [1]. Conceptually, both TEN and the Stevens-Johnson syndrome (SJS) are likely part of the same continuum of clinical presentations [2-6], and they are regarded to be likely distinct from erythema multiforme [7-9]. The precise TEN pathomechanism remains unclear $[1,5,10-12]$. Some toxic metabolites and/or cytotoxic inflammatory cells induce epithelial apoptosis and necrosis [5, 13-15]. Cytotoxic lymphocytes, regulatory $\mathrm{T}$ cells (Treg), macrophages, and dermal dendrocytes (DD) are likely involved, and they probably represent more than passive bystanders [16-20].

Graft-versus-host reaction (GVHR), both in its acute and chronic stages, is responsible for both potentially severe morbidity and mortality [21, 22]. GVHR remains quite frequent in susceptible groups of patients as about half of recipients of allogeneic hematopoietic cell transplantation (HCT) develop GVHR [23-25]. This condition results from a complex and intricate pathobiology sustained by interactions between the donor and host innate and adaptive immune responses. A number of lymphocyte subsets (naive, memory, Treg, Th1, Th17, NK, ...) are involved, as well as eosinophils, mesenchymal stem cells and the monocyte-macrophage lineage including Factor XIIIa+ DD [26-30].

There is an overlap in a series of histopathological signs between early GVHR and various other posttransplantation diseases including some viral exanthems, immune reconstitution rash, and drug reactions [22]. The key points are keratinocyte apoptosis and satellite cell necrosis [31-35]. In absence of specific GVHR histopathological features, the value of skin microscopy remained fairly limited for landing support to the GVHR diagnosis or for ruling out other unrelated diseases. However, targeted immunohistochemistry was 
TABLE 1: Patient demography.

\begin{tabular}{lccc}
\hline Parameter & GVHR & TEN & PTTB \\
\hline M/F & $10 / 7$ & $6 / 11$ & $9 / 8$ \\
Age, years (mean \pm SD) & $35 \pm 8$ & $39 \pm 6$ & $37 \pm 10$ \\
\hline
\end{tabular}

occasionally reported to bring some decisive clues [26, 36$42]$. Both cell differentiation and tensegrity (shape) are better highlighted using some relevant immunopathological markers. It remains that some patients with GVHR develop a drug-induced TEN or a GVHR-related TEN-like lesion [43-46]. In some instances, the histopathological distinction between both conditions remains notoriously difficult or impossible to establish [27].

The aim of the present retrospective study was to revisit the input of immunopathology in the diagnosis of and distinction between cutaneous GVHR and TEN. The study was focused on the histopathology of incipient erythematous GVHR and TEN lesions with epidermal necrosis. Recent partial thickness thermal burns (PTTB) were used as control for epidermal destruction following physical injury.

\section{Subjects and Methods}

The study was approved by the Liège University Hospital and the local Ethics Committee of the Percy Military Teaching Hospital in Clamart. Three series of 17 lesional skin biopsies collected from untreated patients were retrieved from our files. They had been diagnosed as GVHR, TEN, and PTTB, respectively (Table 1). In each case, the epidermis was still present and in close contact with the dermis. Sections $(5 \mu \mathrm{m}$ thick) were cut from formalin-fixed paraffin-embedded biopsies and stained by hematoxylin and eosin. Since clues from histopathology frequently fail to discriminate with confidence cutaneous GVHR from SCAR, immunohistochemistry targeting inflammatory cells, keratinocytes, and dermal cells was used aiming at better discriminating the three conditions. Paraffin sections were used for the avidinbiotin peroxidase method. After a 1-hour incubation time with each of the 15 primary antibodies (Table 2), slides were washed in Tris-buffered saline (TBS) and incubated for $30 \mathrm{~min}$ with the secondary antibody (biotinylated swine anti-rabbit, 1:300, Dakopatts). Slides were rinsed in TBS and covered by the EnVision (Dakopatts, Glostrup, Denmark) polymer-based revelation system. After TBS washing, Fast Red (Dakopatts) was used as chromogen substrate. The last steps consisted of counterstaining with Mayer's hemalum. Negative immunohistochemical controls were performed by omitting or substituting the primary and the secondary antibodies of the laboratory procedure.

Quantitative assessments were performed using computerized image analysis (MOP videoplan Kontron, Eching, Germany). Assessments were performed in a band $0.6 \mathrm{~mm}$ thick of the most superficial part of the skin. Cell counts were expressed as medians per $\mathrm{mm}^{2}$ of tissue (epidermis or dermis) section. Statistical comparisons were performed between TEN and GVHR using the unpaired nonparametric Mann-Whitney $U$ test. Similar comparisons were performed
TABLE 2: Panel of antibodies.

\begin{tabular}{lcc}
\hline Antigen/antibody & Dilution & Source \\
\hline CD4 & $1: 25$ & Dako \\
CD8 & $1: 25$ & Dako \\
CD34 & $1: 50$ & Becton Dickinson \\
CD44 variant 3 & $1: 10$ & Menarini \\
CD45RO & $1: 100$ & Dako \\
CD68 & $1: 200$ & Dako \\
Cytokeratin 15 & $1: 1$ & Neomarkers \\
Factor XIIIa & $1: 100$ & Neomarkers \\
Glutathione S transferase $\pi$ & $1: 100$ & Castra Lab. \\
Ki-67 & $1: 100$ & Dako \\
Lysozyme & $1: 300$ & Dako \\
Mac 387 & $1: 400$ & Dako \\
Myeloperoxidase & $1: 1000$ & Dako \\
Ulex europaeus agglutinin-1 & $1: 2000$ & Sigma \\
Versican & $1: 500$ & Seikagaku Corp. \\
\hline
\end{tabular}

in each condition to assess the relative densities of lymphocytes and macrophages. A $P$ value lower than 0.05 was considered statistically significant.

\section{Results}

3.1. Lymphocytes. $\mathrm{T}$ lymphocytes were scanty in the three conditions. Skin-infiltrating lymphocytes in GVHR were memory CD45RO+ T lymphocytes, as well as cells of the $\mathrm{CD} 4+$ and CD8+ lineages. T lymphocytes grossly exhibited similar patterns of distribution in TEN and GVHR. A majority of them were present along the dermoepidermal junction, and few of them were present inside the epidermis. These latter cells were predominantly CD8+ lymphocytes, whereas the dermal inflammatory infiltrate was predominantly composed of CD4+ cells. By contrast, rare T lymphocytes were identified in recent PTTB. They were predominantly clustered in a perivascular distribution.

The lymphocyte density showed large interindividual differences in the three conditions (Table 3). No significant difference was yielded between TEN and GVHR.

3.2. Macrophages. Mac 387+, CD68+, myeloperoxidase+, and lysozyme+ macrophages were present in both TEN and GVHR lesions (Table 3). They were located along the dermoepidermal junction and haphazardly dispersed in both the dermis and epidermis. By contrast, these cells were rare in PTTB.

In each condition, the Mac 387+ macrophage density was significantly $(P<0.01)$ superior to the CD45RO+ lymphocytes.

3.3. Dermal Resident Cells. Factor XIIIa+ DD appeared numerous and plump in the superficial dermis of most TEN and GVHR (Table 3). By contrast, PTTB did not apparently alter the Factor XIIIa+ DD population. Of note, the CD34+ DD remained tiny and rare in the three conditions. 
TABLE 3: Medians (ranges) of cell densities $/ \mathrm{mm}^{2}$ in the epidermis and superficial dermis of TEN, acute GVHR, and PTTB.

\begin{tabular}{lccc}
\hline Cells/marker & TEN & GVHR & PTTB \\
\hline Dermal T lymphocytes & & & \\
CD45RO & $8(2-19)$ & $13(9-29)$ & $5(1-9)$ \\
CD4 & $4(0-10)$ & $9(5-16)$ & $2(0-6)$ \\
CD8 & $2(0-5)$ & $3(0-6)$ & $1(0-3)$ \\
Dermal macrophages & & & \\
Mac 387 & $17(9-31)$ & $19(13-38)$ & $6(1-8)$ \\
CD68 & $6(3-10)$ & $9(5-17)$ & $1(0-4)$ \\
Myeloperoxidase & $4(3-9)$ & $3(2-8)$ & $0(0-3)$ \\
Lysozyme & $5(3-8)$ & $10(3-16)$ & $1(0-5)$ \\
Dermal dendrocytes & & & \\
Factor XIIIa & $48(24-65)$ & $61(45-83)$ & $52(33-68)$ \\
CD34 & $9(6-15)$ & $12(8-17)$ & $12(5-16)$ \\
Versican & $15(7-32)$ & $19(14-42)$ & $8(3-17)$ \\
Keratinocytes & & & \\
Mac 387 & $389(206-551)$ & $296(157-319)$ & $29(20-39)$ \\
UEA-1 & $97(33-197)$ & $78(12-94)$ & $33(6-81)$ \\
CD44 var 3 & $408(396-569)$ & $333(315-378)$ & $15(12-218)$ \\
GST $\pi$ & $32(0-95)$ & $15(3-61)$ & $0(0-4)$ \\
Ki67 & $6(0-19)$ & $9(7-42)$ & $15(0-63)$ \\
CK15 & $3(0-8)$ & $1(0-4)$ & $4(0-7)$ \\
\hline
\end{tabular}

Versican filled up most dermal cells in TEN and GVHR. They appeared plump compared to those in PTTB. In each condition, they were evenly distributed in the superficial dermis.

3.4. Keratinocytes. L1-protein+ (Mac 387+) keratinocytes were present in all cases of TEN, GVHR, and PTTB irrespective of the histopathologic grades. The cytoplasmic staining was focal or diffuse throughout the epidermis layers, even when the dermal inflammatory infiltrate was scant. Similarly, an uneven to strong Mac 387+ immunolabeling of keratinocytes was present in all biopsies of TEN-altered skin. The L1-antigen was, however, expressed mainly in suprabasal layers of TEN epidermis, with more discrete involvement of basal keratinocytes. The majority of samples from apparently uninvolved skin in TEN patients expressed the L1-antigen in a patchy pattern inside the epidermis, at sites where inflammatory cells were scanty or absent. In PTTB, all keratinocytes showed an intense L1-antigen labeling.

Ulex europaeus agglutinin UEA type I labeled the upper portion or the whole epidermis in the three conditions.

The CD44 var 3 immunoreactivity surrounded each keratinocyte and was not altered by any of the assessed skin conditions.

Glutathione $S$ transferase (GST) $\pi$ was disclosed in small clusters of superficial keratinocytes in TEN.
The Ki-67 labeling was absent or very low $(<2 \%$ basal cells) in the three conditions exhibiting large areas of keratinocyte necrosis.

The cytokeratin $(\mathrm{CK}) 15$ was disclosed in rare $(<1 \%)$ basal cells.

\section{Discussion}

TEN is one of the most dramatic drug-induced SCAR [1]. This life-threatening disease is characterized by the extensive destruction of the epidermis and epithelial mucosae. Some clues suggest that TEN results from a specific alteration of drug metabolism in keratinocytes [11, 15, 47]. More than 100 different drugs are currently involved in TEN, but only a minority of them accounts for the vast majority of cases [8]. Molecular and morphologic features of apoptosis were demonstrated in TEN-involved keratinocytes during the initial stage of the disease [13-15]. The following phase of TEN is characterized by full-thickness epidermal necrosis. Hence, it is assumed that the TEN pathomechanism likely combines early apoptosis and late necrosis $[13,14]$.

GVHR is recognized by clinicopathological alterations in recipients of HCT or bone marrow transplantation [21, $22]$. Both immunological and nonspecific phenomena contribute to the clinical aspects. GVHR is one of the major complications of HCT and is responsible for posttherapeutic morbidity, decreased quality of life, and mortality $[21,22]$. GVHR is critically induced and maintained by donor immunocompetent cells that are particularly directed against epithelia showing fast renewal including the liver, gastrointestinal tract, and epidermis.

In some instances, a clinicopathological overlap exists between aspects of GVHR and TEN. This mixed condition typically represents a puzzling diagnostic dilemma $[27,44,46]$. Histopathology of both conditions shows a sparse inflammatory cell infiltrate with keratinocyte apoptosis. Satellite cell necrosis has been thought to be a typical feature of GVHR, although the same aspect has similarly been recorded in some TEN cases.

Until recently, macrophages were unfrequently reported in TEN and GVHR, and they were not considered to play a pivotal role in the disease. However, biopsies from the liver, gut, and skin of patients with lethal GVHR showed a striking preponderance of CD68+ macrophages in the inflammatory infiltrate. They were variably reported to be more or less numerous than $\mathrm{T}$ lymphocytes. The macrophage preponderance was especially found in the most severely damaged skin areas [27]. Indeed, the monocyte/macrophage L1-protein (Mac 387) expression was reported in over $80 \%$ of GVHR, but their possible primary or secondary involvement still remains debated. The preponderant macrophage infiltrate occasionally observed might result from the antilymphocyte effect achieved by preventive immunosuppressive treatments that generally do not target macrophages. However, this possibility does not rule out that primary macrophagemediated GVHR exists. In such instance, GVHR resembles the inflammatory reaction to imiquimod application of the skin [48]. 
Macrophages could tentatively serve as a clue distinguishing TEN and GVHR. When the immunosuppressive regimen to HSCT fails to perform adequately, cutaneous GVHR shows a predominance of T lymphocytes over macrophages. Nowadays, the histopathological presentation of GVHR has changed, with refinements in the biologic and pharmacologic prevention of HSCT adverse effects. As immunosuppressive regimen preferentially targets $\mathrm{T}$ lymphocytes, the inflammatory reaction becomes restricted to Mac 387+ macrophages. The latter cells always outnumber the CD68+, myeloperoxidase+, and lysozyme+ macrophages. They are at least as numerous as the combination of CD45RO+, CD4+, and CD8+ T lymphocytes in TEN and even more abundant in recent cases. Even when macrophages outnumber $\mathrm{T}$ lymphocytes in TEN, a pure macrophage infiltrate is never seen. Hence, an inflammatory infiltrate composed almost exclusively of Mac 387+ macrophages could be a clue for a lymphocyte-abated GVHR.

Factor XIIIa+ DD encompass distinct functional subtypes [49]. Their numbers in the superficial dermis are variable and appear increased in the vast majority of TEN cases [18]. The large number of Factor XIIIa+ DD and their plump appearance in the perilesional and lesional TEN skin suggest their activation during the initial steps of the disease. The CD34+ DD do not show similar stimulation signs. The Factor XIIIa+ DD ultrastructural aspects are similar in TEN and GVHR, showing enlarged endoplasmic reticulum, and phagocytosis of collagen fibres and mast cell granules [39]. It is inferred that both the number and aspect of DD do not help discriminating the two diseases. They provide an additional link between them. Similarly, the versican+ dendritic cells appear stimulated in TEN and GVHR.

Keratinocytes are clearly the main target cells in both TEN and GVHR. Several immunohistochemical markers identifying keratinocytes and their adhesion molecules have been proposed to reveal changes in the epidermis of these two conditions [27]. The $365-\mathrm{kDa}$ L1-protein exhibits some antimicrobial properties. The name calprotectin was coined for it. It is specifically identified using the Mac 387 monoclonal antibody during routine immunostaining. It consists of three calcium noncovalently bound polypeptide chains. It is expressed in neutrophils, monocytes, and some reactive macrophages, as well as in mucosal epithelium and reactive epidermis. Keratinocytes in the vicinity of inflammatory cells or covering tumors frequently express the L1-protein, a condition which has been interpreted as a nonspecific marker of cellular stress. The L1-protein keratinocyte expression during GVHR probably discloses sublethal cell injury. Such a finding appears to be very sensitive, although it lacks specificity. Hence, epidermal L1-protein expression is not specific and cannot be used for distinguishing GVHR from TEN.

In the present study, immunohistochemistry directed to UEA-1, CD44 var 3, and CK15 did not reveal marked differences between TEN and GVHR keratinocytes. The number of Ki67+ keratinocytes engaged in cell proliferation was similar in these two conditions. By contrast, it was largely overexpressed in some PTTB cases engaged in a repair phase. By contrast, the GST $\pi$ immunolabeling was more frequently overexpressed in TEN than in GVHR, and it was often absent in PTTB.

\section{Conclusion}

TEN, GVHR, and PTTB share in common extensive necrosis of the full-thickness epidermis. The induction mechanisms are strikingly distinct, involving a drug cytotoxic reaction in TEN, a lymphocyte-mediated destruction in GVHR, and a physical threat in PTTB. The clinicopathological differential diagnosis between TEN and acute GVHR is blurred by overlaps in the skin conditions. This retrospective study suggests that the macrophage/lymphocyte balance represents a clue for distinguishing both conditions.

\section{Acknowledgments}

This work was supported by a grant from the Fonds d'Investissement de la Recherche Scientifique of the University Hospital of Liège. No other sources of funding were used to assist in the preparation of this paper. The authors have no conflict of interests that is directly relevant to the content of this paper. The authors appreciate the excellent secretarial assistance of Ida Leclercq.

\section{References}

[1] G. E. Piérard and P. Paquet, "Facing up to toxic epidermal necrolysis," Expert Opinion on Pharmacotherapy, vol. 11, no. 15, pp. 2443-2446, 2010.

[2] P. O. Fritsch and A. Sidoroff, "Drug-induced Stevens-Johnson syndrome/toxic epidermal necrolysis," American Journal of Clinical Dermatology, vol. 1, no. 6, pp. 349-360, 2000.

[3] R. Abe, T. Shimizu, A. Shibaki, H. Nakamura, H. Watanabe, and H. Shimizu, "Toxic epidermal necrolysis and Stevens-Johnson syndrome are induced by soluble fas ligand," American Journal of Pathology, vol. 162, no. 5, pp. 1515-1520, 2003.

[4] B. Khalili and S. L. Bahna, "Pathogenesis and recent therapeutic trends in Stevens-Johnson syndrome and toxic epidermal necrolysis," Annals of Allergy, Asthma and Immunology, vol. 97, no. 3, pp. 272-281, 2006.

[5] A. T. Borchers, J. L. Lee, S. M. Naguwa, G. S. Cheema, and M. E. Gershwin, "Stevens-Johnson syndrome and toxic epidermal necrolysis," Autoimmunity Reviews, vol. 7, no. 8, pp. 598-605, 2008.

[6] M. Mockenhaupt, "The current understanding of StevensJohnson syndrome and toxic epidermal necrolysis," Expert Review of Clinical Immunology, vol. 7, no. 6, pp. 803-813, 2011.

[7] R. Forman, G. Koren, and N. H. Shear, "Erythema multiforme, Stevens-Johnson syndrome and toxic epidermal necrolysis in children: a review of 10 years' experience," Drug Safety, vol. 25, no. 13, pp. 965-972, 2002.

[8] R. Watanabe, H. Watanabe, C. Sotozono et al., "Critical factors differentiating erythema multiforme majus from StevensJohnson syndrome (SJS)/toxic epidermal necrolysis (TEN)," European Journal of Dermatology, vol. 21, no. 6, pp. 889-894, 2011. 
[9] S. Iwai, H. Sueki, H. Watanabe et al., "Distinguishing between erythema multiforme major and Stevens-Johnson syndrome/toxic epidermal necrolysis immunopathologically," Journal of Dermatology, vol. 39, no. 9, pp. 781-786, 2012.

[10] F. A. Pereira, A. V. Mudgil, and D. M. Rosmarin, "Toxic epidermal necrolysis," Journal of the American Academy of Dermatology, vol. 56, no. 2, pp. 181-200, 2007.

[11] P. Paquet and G. E. Piérard, "New insights in toxic epidermal necrolysis (lyells syndrome): clinical considerations, pathobiology and targeted treatments revisited," Drug Safety, vol. 33, no. 3, pp. 189-212, 2010.

[12] A. Downey, C. Jackson, N. Harun et al., "Toxic epidermal necrolysis: review of pathogenesis and management," Journal of the American Academy of Dermatology, vol. 66, no. 6, pp. 9951003, 2012.

[13] C. Paul, P. Wolkenstein, H. Adle et al., "Apoptosis as a mechanism of keratinocyte death in toxic epidermal necrolysis," British Journal of Dermatology, vol. 134, no. 4, pp. 710-714, 1996.

[14] P. Paquet and G. E. Pierard, "Toxic epidermal necrolysis: revisiting the tentative link between early apoptosis and late necrosis (Review)," International Journal of Molecular Medicine, vol. 19, no. 1, pp. 3-10, 2007.

[15] P. Paquet, P. Delvenne, and G. E. Piérard, "Drug interactions with normal and TEN epidermal keratinocytes," Current Drug Safety, vol. 7, no. 5, pp. 352-356, 2012.

[16] P. Paquet, A. Nikkels, J. E. Arrese, A. Vanderkelen, and G. E. Pierard, "Macrophages and tumor necrosis factor $\alpha$ in toxic epidermal necrolysis," Archives of Dermatology, vol. 130, no. 5, pp. 605-608, 1994.

[17] W. H. Chung, S. I. Hung, J. Y. Yang et al., "Granulysin is a key mediator for disseminated keratinocyte death in StevensJohnson syndrome and toxic epidermal necrolysis," Nature Medicine, vol. 14, no. 12, pp. 1343-1350, 2008.

[18] P. Paquet, P. Quatresooz, and G. E. Piérard, "Factor-XIIIapositive dendrocytes in drug-induced toxic epidermal necrolysis (Lyell's syndrome): paradoxical activation in skin and rarefaction in lymph nodes," Dermatology, vol. 206, no. 4, pp. 374-378, 2003.

[19] P. Paquet, D. de Groote, and G. E. Piérard, "Functionally active macrophage-derived myeloperoxidase in the skin of druginduced toxic epidermal necrolysis," Dermatology, vol. 220, no. 3, pp. 201-207, 2010.

[20] M. Tohyama, H. Watanabe, S. Murakami et al., "Possible involvement of CD14+ CD16+ monocyte lineage cells in the epidermal damage of Stevens-Johnson syndrome and toxic epidermal necrolysis," British Journal of Dermatology, vol. 166, no. 2, pp. 322-330, 2012.

[21] D. Couriel, H. Caldera, R. Champlin, and K. Komanduri, "Acute graft-versus-host disease: pathophysiology, clinical manifestations, and management," Cancer, vol. 101, no. 9, pp. 1936-1946, 2004.

[22] P. Häusermann, R. B. Walter, J. Halter et al., "Cutaneous graftversus-host disease: a guide for the dermatologist," Dermatology, vol. 216, no. 4, pp. 287-304, 2008.

[23] M. Mielcarek, P. J. Martin, W. Leisenring et al., "Graftversus-host disease after nonmyeloablative versus conventional hematopoietic stem cell transplantation," Blood, vol. 102, no. 2, pp. 756-762, 2003.

[24] J. V. Schaffer, "The changing face of graft-versus-host disease," Seminars in Cutaneous Medicine and Surgery, vol. 25, no. 4, pp. 190-200, 2006.
[25] S. Paczesny, D. Hanauer, Y. Sun, and P. Reddy, "New perspectives on the biology of acute GVHD," Bone Marrow Transplantation, vol. 45, no. 1, pp. 1-11, 2010.

[26] Y. H. Yoo, B. S. Park, D. Whitaker-Menezes, R. Korngold, and G. F. Murphy, "Dermal dendrocytes participate in the cellular pathology of experimental acute graft-versus-host disease," Journal of Cutaneous Pathology, vol. 25, no. 8, pp. 426-434, 1998.

[27] P. Paquet, J. E. Arrese, Y. Beguin, and G. E. Piérard, "Clinicopathological differential diagnosis of drug-induced toxic epidermal necrolysis (Lyell's syndrome) and acute graft-versushost reaction," Current Topics in Pathology, vol. 94, pp. 49-63, 2001.

[28] A. D. Hess, "Modulation of graft-versus-host disease: role of regulatory T lymphocytes," Biology of Blood and Marrow Transplantation, vol. 12, no. 2, pp. 13-21, 2006.

[29] D. Noguchi, D. Wakita, T. Ohkuri et al., "Blockade of IL-6signaling inhibits the pathogenesis of CD4+ T cell-mediated lethal graft-versus-host reaction against minor histocompatibility antigen," Immunology Letters, vol. 136, no. 2, pp. 146-155, 2011.

[30] V. R. Sharon, T. H. Konia, K. L. Barr et al., "Assessment of the "no eosinophils" rule : are eosinophils truly absent in pityriasis lichenoides, connective tissue disease, and graft-vshost disease?" Journal of Cutaneous Pathology, vol. 39, no. 4, pp. 413-418, 2012.

[31] A. C. Gilliam, D. Whitaker-Menezes, R. Korngold, and G. F. Murphy, "Apoptosis is the predominant form of epithelial target cell injury in acute experimental graft-versus-host disease," Journal of Investigative Dermatology, vol. 107, no. 3, pp. 377-383, 1996.

[32] D. Whitaker-Menezes, S. C. Jones, T. M. Friedman, R. Korngold, and G. F. Murphy, "An epithelial target site in experimental graft-versus-host disease and cytokine-mediated cytotoxicity is defined by cytokeratin 15 expression," Biology of Blood and Marrow Transplantation, vol. 9, no. 9, pp. 559-570, 2003.

[33] C. C. Hofmeister, A. Quinn, K. R. Cooke, P. Stiff, B. Nickoloff, and J. L. M. Ferrara, "Graft-versus-host disease of the skin: life and death on the epidermal edge," Biology of Blood and Marrow Transplantation, vol. 10, no. 6, pp. 366-372, 2004.

[34] J. L. M. Ferrara, K. R. Cooke, T. Teshima et al., "The pathophysiology of graft-versus-host disease," in Graft-Versus-Host Disease, J. Ferrara, K. Cooke, and H. J. Deeg, Eds., pp. 1-34, Dekker, New York, NY, USA, 2005.

[35] Q. Zhan, S. Signoretti, D. Whitaker-Menezes, T. M. Friedman, R. Korngold, and G. F. Murphy, "Cytokeratin15-positive basal epithelial cells targeted in graft-versus-host disease express a constitutive antiapoptotic phenotype," Journal of Investigative Dermatology, vol. 127, no. 1, pp. 106-115, 2007.

[36] S. Kohler, M. R. Hendrickson, N. J. Chao, and B. R. Smoller, "Value of skin biopsies in assessing prognosis and progression of acute graft-versus-host disease," American Journal of Surgical Pathology, vol. 21, no. 9, pp. 988-996, 1997.

[37] G. E. Piérard, N. Nikkels-Tassoudji, A. F. Nikkels et al., "Epidermal calprotectin expression in lymphocyte-depleted cutaneous graft-versus-host reaction," Archives of Argentinos Dermatology, vol. 48, pp. 139-142, 1998.

[38] G. E. Pierard, N. Nikkels-Tassoudji, J. E. Arrese, A. F. Nikkels, T. H. Le, and C. P. Franchimont, "L1-protein in incipient lymphocyte-depleted graft-versus-host disease. Expression in keratinocytes and coexpression with factor XIIIa in dermal cells," Turkish Journal of Dermatopathology, vol. 7, no. 1-2, pp. 10-13, 1998. 
[39] T. Hermanns-Lê, P. Paquet, C. Piérard-Franchimont, J. E. Arrese, and G. E. Piérard, "Regulatory function of factor-XIIIapositive dendrocytes in incipient toxic epidermal necrolysis and graft-versus-host reaction. A hypothesis," Dermatology, vol. 198, no. 2, pp. 184-186, 1999.

[40] Y. Zhou, M. J. Barnett, and J. K. Rivers, "Clinical significance of skin biopsies in the diagnosis and management of graft-vs-host disease in early postallogeneic bone marrow transplantation," Archives of Dermatology, vol. 136, no. 6, pp. 717-721, 2000.

[41] D. Heldal, L. Brinch, S. A. Evensen et al., "Skin biopsies for early diagnosis and prognosis of graft-versus-host disease in recipients of allogeneic stem cells from blood or bone marrow," Bone Marrow Transplantation, vol. 34, no. 4, pp. 345-350, 2004.

[42] A. T. Bridge, R. P. Nelson, J. E. Schwartz, G. W. Mirowski, and S. D. Billings, "Histological evaluation of acute mucocutaneous graft-versus-host disease in nonmyeloablative hematologic stem cell transplants with an observation predicting an increased risk of progression to chronic graft-versus-host disease," American Journal of Dermatopathology, vol. 29, no. 1, pp. 1-6, 2007.

[43] C. Merle, D. Blanc, M. Flesch et al., "A picture of epidermal necrolysis after hepatic allograft. Etiologic aspects," Annales of Dermatology and Venereology, vol. 117, no. 9, pp. 635-639, 1990.

[44] G. Villada, J. C. Roujeau, C. Cordonnier et al., “Toxic epidermal necrolysis after bone marrow transplantation: study of nine cases," Journal of the American Academy of Dermatology, vol. 23, no. 5, pp. 870-875, 1990.

[45] H. Takeda, Y. Mitsuhashi, S. Kondo, Y. I. Kato, and K. Tajima, "Toxic epidermal necrolysis possibly linked to hyperacute graftversus- host disease after allogeneic bone marrow transplantation," Journal of Dermatology, vol. 24, no. 10, pp. 635-641, 1997.

[46] N. Stone, S. Sheerin, and S. Burge, “Toxic epidermal necrolysis and graft vs. host disease: a clinical spectrum but a diagnostic dilemma," Clinical and Experimental Dermatology, vol. 24, no. 4, pp. 260-262, 1999.

[47] F. P. Nestel, K. S. Price, T. A. Seemayer, and W. S. Lapp, "Macrophage priming and lipopolysaccharide-triggered release of tumor necrosis factor $\alpha$ during graft-versus-host disease," Journal of Experimental Medicine, vol. 175, no. 2, pp. 405-413, 1992.

[48] T. Hermanns-Lê, P. Paquet, A. F. Nikkels, C. PiérardFranchimont, and G. E. Piérard, "Prolonged imiquimod treatment and graft-versus-host reaction: histological mimicry in the skin infiltration pattern of the monocyte-macrophagedendrocyte lineage," Dermatology, vol. 206, no. 4, pp. 361-365, 2003.

[49] P. Quatresooz, P. Paquet, T. Hermanns-Lê, and G. E. Piérard, "Molecular mapping of factor XIIIa-enriched dendrocytes in the skin (review)," International Journal of Molecular Medicine, vol. 22, no. 4, pp. 403-409, 2008. 


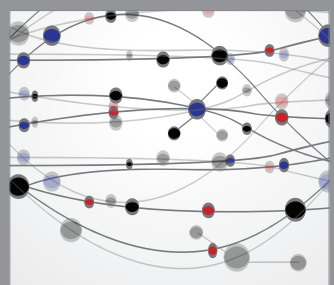

The Scientific World Journal
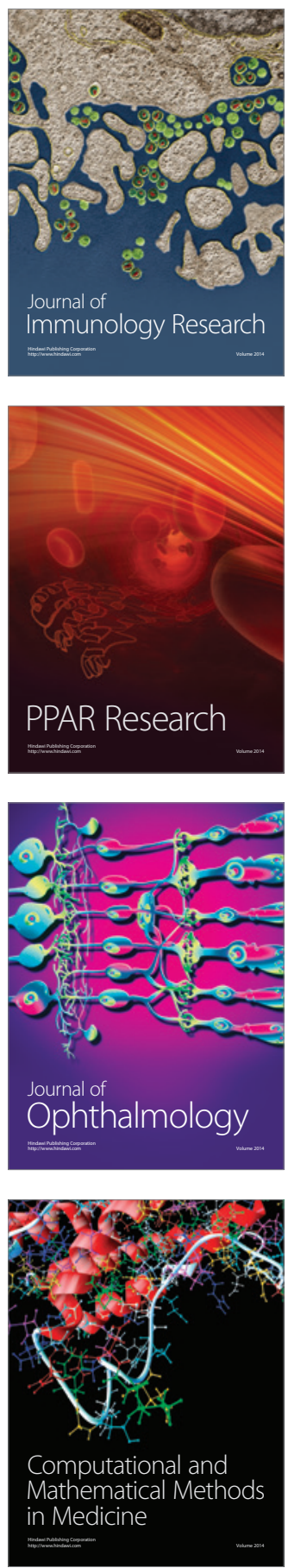

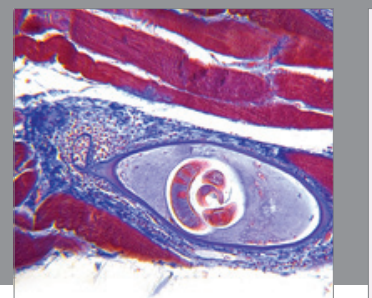

Gastroenterology

Research and Practice
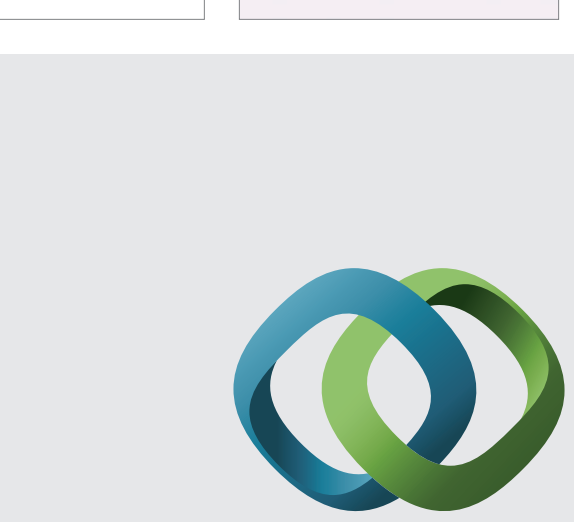

\section{Hindawi}

Submit your manuscripts at

http://www.hindawi.com
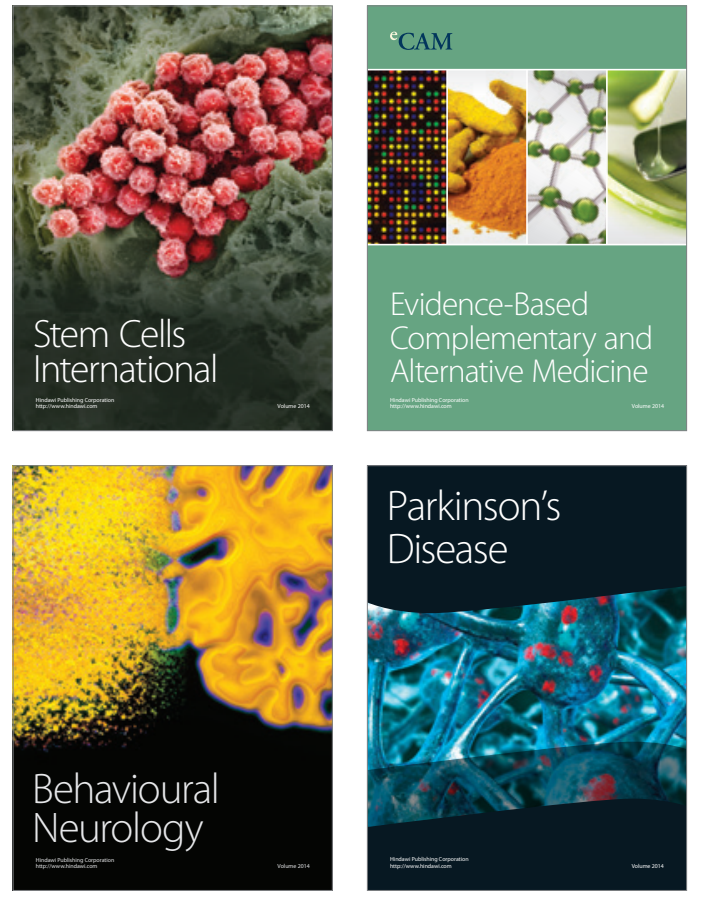
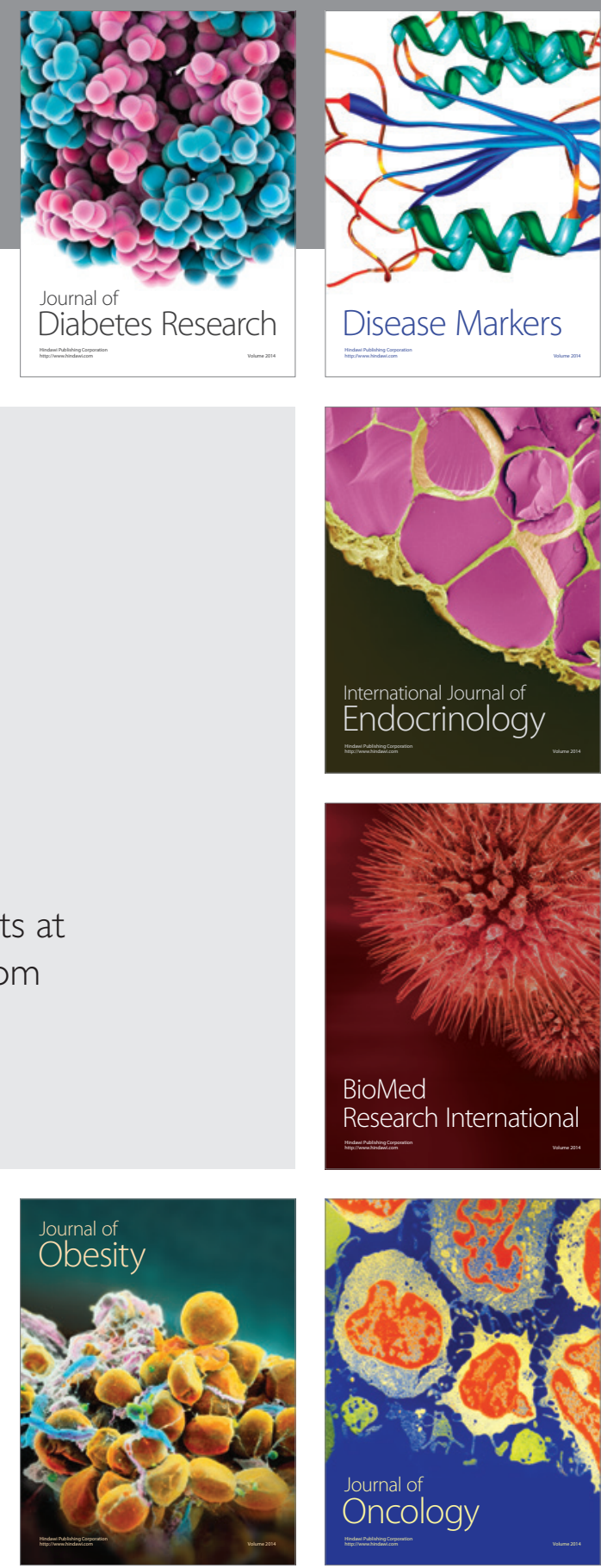

Disease Markers
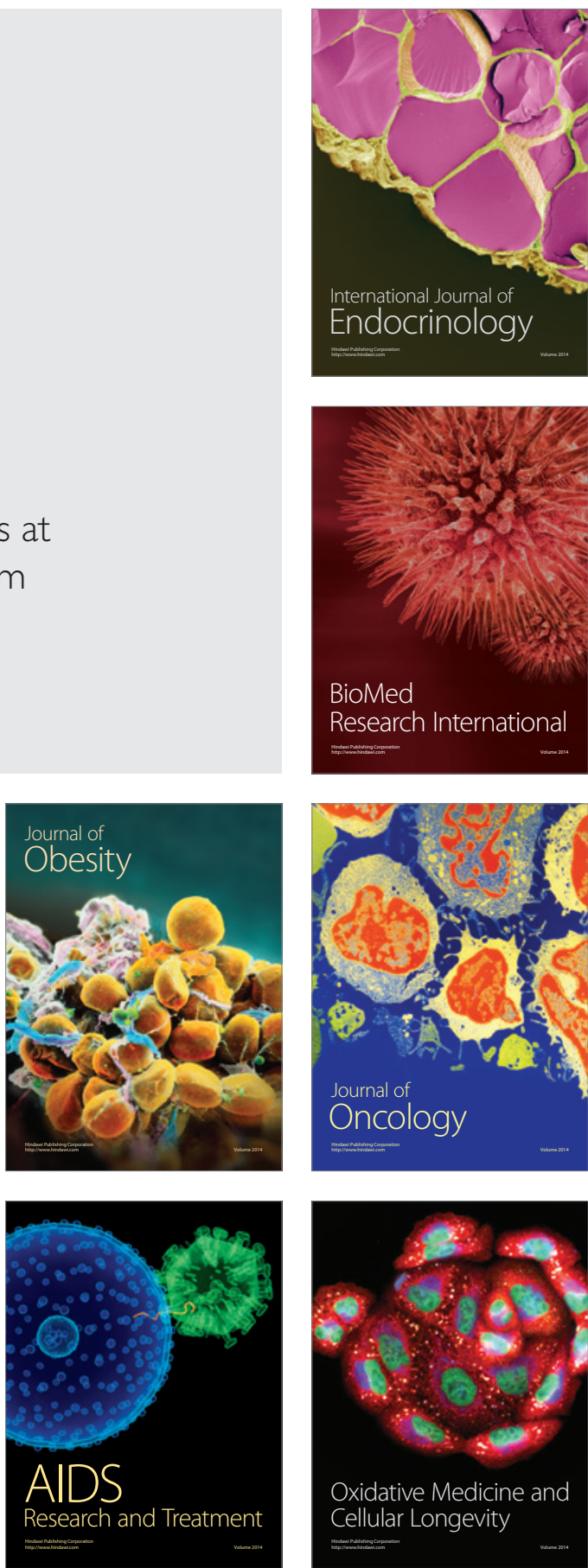